\title{
Nonrespiratory Sinus Arrhythmia
}

\author{
José Barbosa Filho, Paulo Roberto B. Barbosa, Ivan Cordovil
}

Rio de Janeiro, RJ - Brazil

\begin{abstract}
We report here 2 cases of sinus arrhythmia considered to be a form of nonrespiratory sinus arrhythmia because they did not have variances in the RR interval sequence within the oscillations modulated by respiration. Because the patients had pulsus alternans similar that observed in bigeminy, and because they did not have signs or symptoms of heart failure, we believe the arrhythmias represent intrinsic alterations of the electric activity of the sinus node.
\end{abstract}

Respiratory sinus arrhythmia is a normal phenomenon observed more clearly in children and adolescents. In elderly individuals, it becomes imperceptible both clinically and electrocardiographically. Therefore, the variation in the RR intervals modulated by respiration can only be analyzed through digitized systems with filters capable of detecting and properly processing small variations in these intervals. Using algorithms, we observed that the sympathetic-vagal system modulates the heart rate within a frequency band that ranges from $0.1 \mathrm{~Hz}$ to $0.4 \mathrm{~Hz}$, regardless of the clinical picture of the patients.

\section{Case reports}

Patients were studied according to the principles of the Helsinki Declaration and Brazilian laws, and the study protocol was approved by the Medical Ethics Committee of the Instituto Nacional de Cardiologia Laranjeiras (National Institute of Cardiology-Laranjeiras). The patients gave informed consent.

Two individuals were included in the study from the time of their first appointment at the Ambulatory Clinic of the Division of Arterial Hypertension of the National Institute of Cardiology-Laranjeiras. The selection of these pa-

Instituto Nacional de Cardiologia Laranjeiras, Ministério da Saúde e Universidade Gama Filho

Mailing address: José Barbosa Filho - Rua Souza Lima, 324/201 - 22081-010 Rio de Janeiro, RJ tients for the echocardiographic RR oscillation interval study was prompted by the alternans features, both graphic and auscultatory, of the arterial pulsus.

Case 1 - A 79-year-old female presented with atypical complaints for heart disease, characterized by chest pain and tinnitus, and she was taking vitamins and a cerebral vasodilator. Physical and laboratory examinations, as well as radiology and electrocardiography, were within normal limits. The echocardiogram showed signs of aortic and mitral arteriosclero sis, preserved ventricular function, and a $3.4 \mathrm{~cm}$ left atrium diameter. Blood pressure was $127 / 61 \mathrm{mmHg}$ (average $=$ $85 \mathrm{mmHg}$ ), and pulsus alternans was present (fig. 1).

Case 2 - An 80-year-old male presented with a clinical history of stable angina, well controlled with clinical treatment. He was taking oral nitrates and salicylic acids. Radiology showed a discreet enlargement of the cardiac area (cardiothoracic index $=52 \%$ ), and physical and laboratory examinations of the heart were within normal limits. An electrocardiogram showed first-degree atrioventricular block $($ PR interval $=260 \mathrm{~ms})$. Echocardiogram showed signs of aortic valve sclerosis, preserved ventricular function, and a left atrium $3.8 \mathrm{~cm}$ in diameter. Blood pressure was 151/ $81 \mathrm{mmHg}$ (average $=104 \mathrm{mmHg}$ ) and pulsus alternans was present (fig. 1).

Both patients were in sinus rhythm and had no complaints of palpitations.

The electrocardiographic signals of the two patients were acquired during 300s, using the AECG03 amplifier (Lynx Tecnologia Ltda., São Paulo) and analyzed using the ECGAR $^{\circledR}$ system, developed for this purpose and previously described ${ }^{1}$. The signs were acquired with the individuals in supine position and breathing normally, in a quiet place with temperature maintained at $25^{\circ} \mathrm{C}$, during the period between $14 \mathrm{~h}$ and $17 \mathrm{~h}$. The examinations were performed at least 4 hours after the last meal.

Blood pressure was assessed using an oscillograph after the patient had 5-minutes of bed rest in the supine position. During auscultation, pressure curves were recorded during a 25-second interval.

Analysis of the RR variability (V-RR) in both patients was performed in time and frequency domains. With regard to time domain, we assessed the mean heart rate of the nor- 


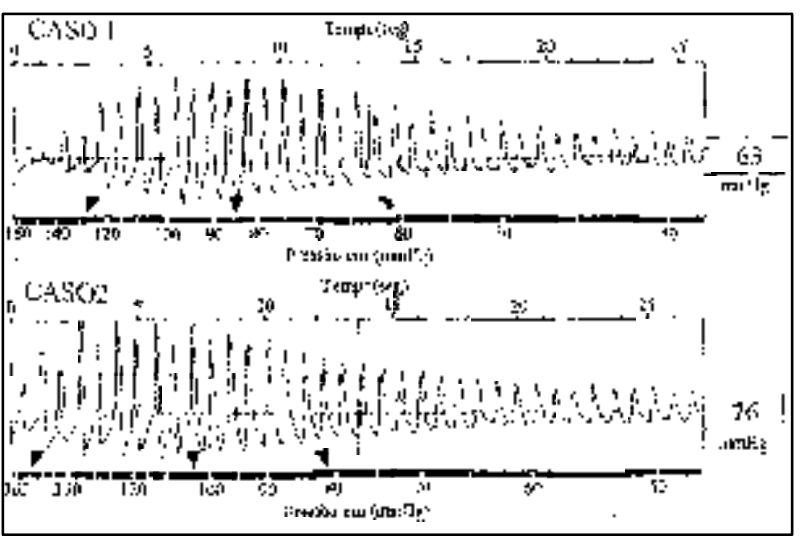

Fig. 1 - Record of pulsus during blood pressure assessment through the oscillometric method. Observe the pulsus alternans.

mal RR intervals series, calculated as the values of the instantaneous cardiac rate. With regard to frequency domain, the series of consecutive normal RR intervals was interpolated, and the power spectrum was calculated using a previously described technique ${ }^{2}$. We analyzed the features of the areas of the power spectrum between 0.05 and $1 \mathrm{~Hz}$, and we identified the dominant peak of power density.

The $\mathrm{P}$ waveform preceding each normal beat was analyzed and compared, 2 by 2 , with the subsequent beats, using a simple correlation.

Both the normal RR intervals and the power spectrum of a control individual of the same age group can be observed in figure 2 .

The distribution of the RR intervals of both patients in the frequency domain (fig. 3) is very different from those assessed in the healthy individual from the same age group (fig. 2). The behavior of the series of normal RR intervals and the distribution of several V-RR bands can be observed in figure 2 . In the patients, the main features are the low energy of the entire power spectrum, especially that from the highfrequency region, and the presence of an unexpected

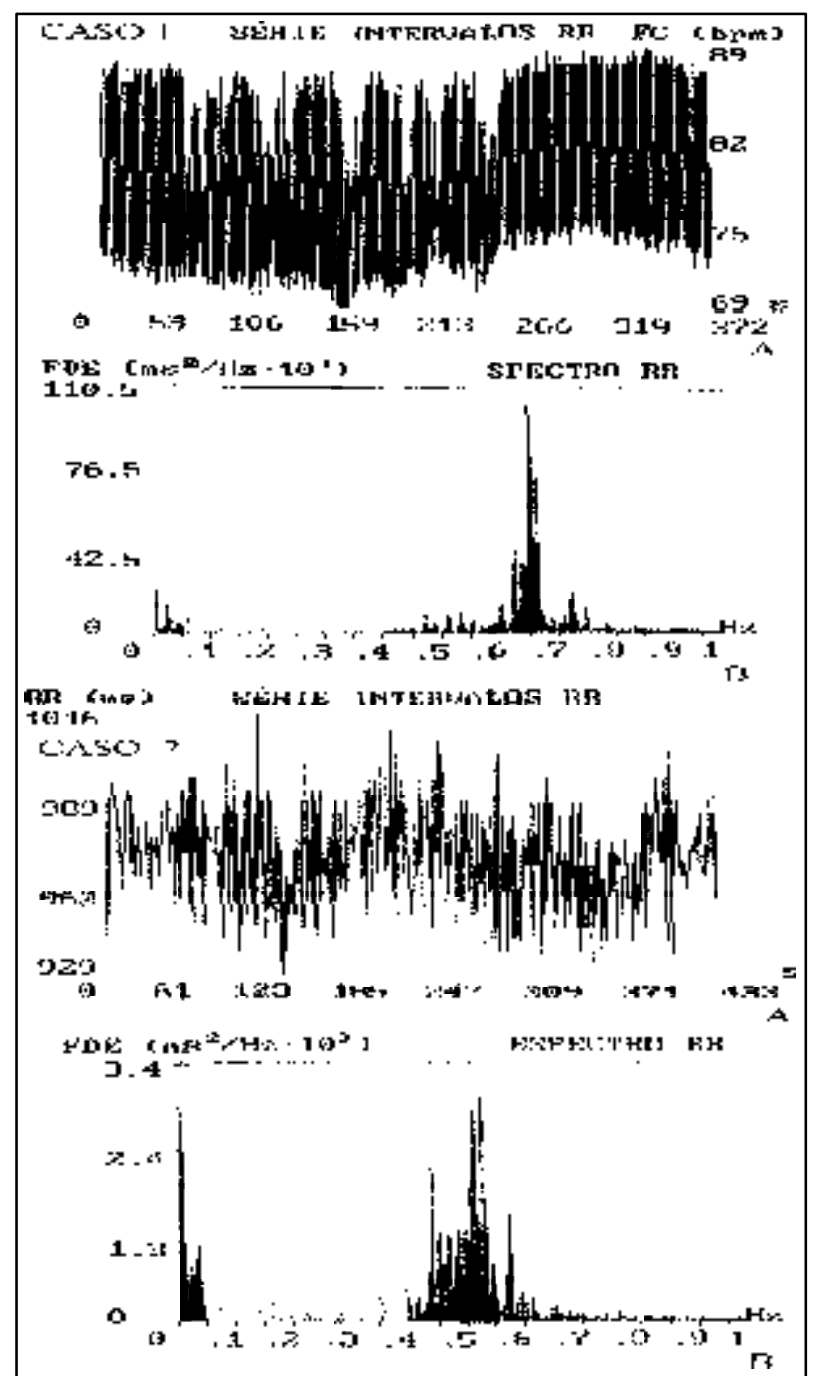

Fig. 3 - Record of the series of the normal RR intervals and the respective power spectrum of the 2 study patients. Observe that the main features of the power spectrum are its low energy and the presence of peak energy in the high-frequency band. Data expressed in the ordinates and abscissas, of both graphs, are the same as those of figure 2. FPD-function of power spectral density.

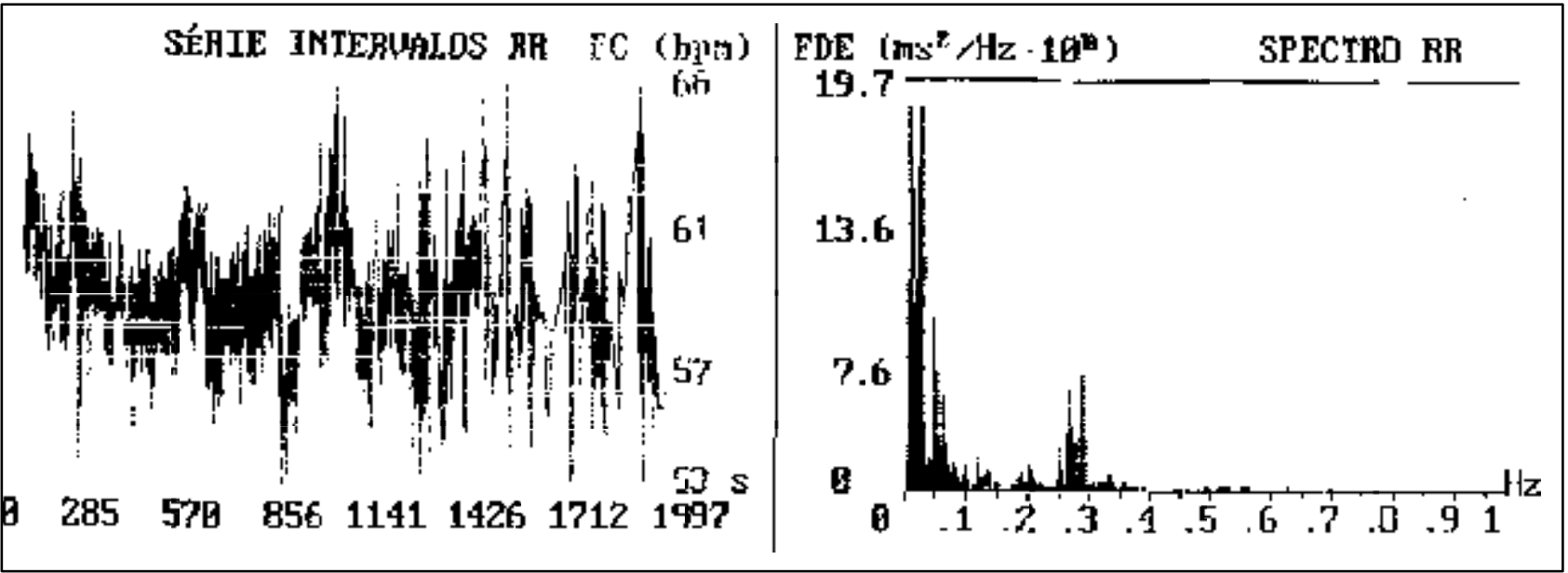

Fig. 2 -Record of the series of normal RR intervals and the respective power of a control individual of the same age group of the assessed patients. Observe that the energy of the spectrum does not go beyond $0.4 \mathrm{~Hz}$. In the ordinates of the graph, RR intervals are displaced: in case 1, they are expressed in bpm; in case 2 , they are expressed in ms. In the abscissa, the time of detecting the signs of the electrocardiogram are expressed in $\mathrm{ms}$. In the FPD graphic, the ordinates represent the values of the spectrum energy, in $\mathrm{ms}^{2} / \mathrm{Hz} .10^{3}$, and the abscissa represent the values of the frequencies, in $\mathrm{Hz}$, of the bands assessed. FPD-function of the power spectral density. 


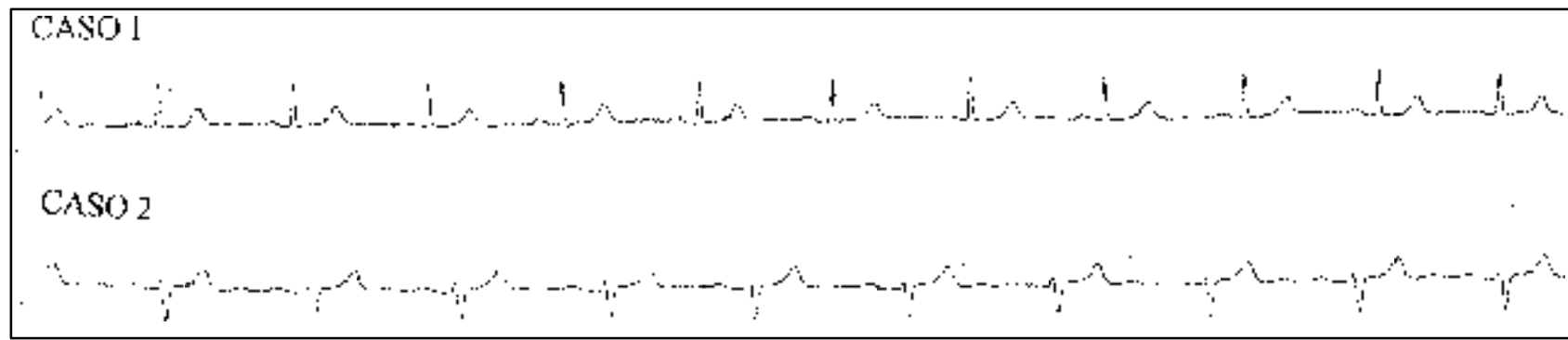

Fig. 4 - Record of the $\mathrm{D}_{2}$ derivation of the electrocardiogram of the study patients, where the maintenance of the sinus mechanism can be observed, $\mathrm{P}$ waves of the same morphology and RR intervals without clear variations.

concentration of energy in the very high-frequency band (fig. 3). The fact that the frequency in $\mathrm{Hz}$, where the energy of the power density spectrum is focused, corresponds to half of the RR interval related to the mean heart rate related to time is remarkable. Thus, in case 1 , the energy of the power spectrum was $0.658 \mathrm{~Hz}$, corresponding to a heart rate of $79 \mathrm{bpm}$, and, in case 2, it was $0.515 \mathrm{~Hz}$, corres ponding to a heart rate of $61.8 \mathrm{bpm}$ (fig. 3 ).

Electrocardiographic recordings (fig. 4) show that the sinus mechanism is maintained, with $P$ waves of the same morphology and a consistent correlation between the waveforms, showing that the behavior of the RR intervals does not correspond to any kind of recognized supraventricular or ventricular arrhythmia.

The record of the arterial pulse curve during assessment of blood pressure is typical of pulsus alternans (fig. 1).

\section{Discussion}

V-RR with the very particular pattern of distribution of the power spectrum frequency, different from those frequently observed in healthy and cardiac patients, was first described by Binkley et al. ${ }^{3}$, in 1 patient with cardiac failure. However, we have not found any description of this characteristic pattern of V-RR in the literature in patients without significant cardiac structural alterations and without arrhythmia, which, if it were, could explain this behavior of the RR intervals ${ }^{4}$. In fact, the behavior of the V$\mathrm{RR}$, both in elderly and cardiac patients, is characterized by low energy and by homogeneous distribution of energy in several bands of the power spectrum ${ }^{2}$. With regards to rapid variations of the normal $\mathrm{RR}$ intervals, the reduction in high-frequency energy is related to a decrease in the activity of the parasympathetic nervous system.

Our patients had, as basic features, great concentrations of energy in the very high-frequency area of the power spectrum, therefore, out of the region usually modulated by the vagal-sympathetic system (fig. 3).

Similarly to that which Binkley et $\mathrm{al}^{3}$ observed, our patients had alterations in the power spectrum, the record of the curve of systemic blood pressure with the typical appearance of pulsus alternans that called attention to the phenomenon (fig. 1), although our patients did not have signs or symptoms of heart failure.

The responsible mechanism for these alterations, as described by Binkley et al $^{1}$, peaks at a very high frequency of the power spectrum associated with pulsus alternans and is related to a reduced baroreflex sensitivity, similar to a lowgain feedback control system in which uncontrolled oscillations in blood pressure signals may occur ${ }^{5}$. Thus, oscillations in blood pressure would cause alterations similar to those of V-RR.

On the other hand, we could suppose that the mechanism was inverted. That is, rapid changes in the V-RR would depend on an electrical alternation in the activity of the sinus node, regardless of respiration. Thus, electromechanical alterations observed would have a mechanism similar to that of bigeminy (shorter RR interval, lower blood pressure), having intrinsic physiopathologic alterations of the sinus node as a base. From this hypothesis, we can postulate that this behavior of the V-RR may represent a form of sinus node disease.

From the evolvement of the patients, we observed that, in a 3-year follow-up, our patients maintained the same pattern, both of the power spectrum and the behavior of the pulse wave, without having clinical alterations worth mentioning.

\section{References}

1. Barbosa PRB. A signal-averaging system for surface ECG signal analysis. Phys Med Biol 1994;39:412.

2. BarbosaPR,BarbosaFJ,MoraisdeSáCA. Influênciadaidade, sexoedoençacoronária sobre amodulaçãoautonômica docoração. ArqBras Cardiol 1996; 67:325-9.

3. Binkley PF, Eaton GM, Nunciata E, et al. Heart rate alternans. Ann Intern Med 1995; 122: 115-7.
4. Frank JL, Ropper AH,Zuniga G. Acute intracranial lesions and respiratory sinus arrhytmia. Arch Neurol 1992; 49: 1200-7.

5. Franklin GF, Powell JD, Emami-Nacini A. Feedback Control of Dynamic Systems. New York: Addisson-Wesley; 1991:111-3. 